\title{
https://doi.org/10.48009/2_iis_2007_107-111 \\ A PICTURE IS WORTH A THOUSAND WORDS: USING DIGITAL STORYTELLING IN THE CLASSROOM
}

\author{
Michele Kleckner, Elon University, mkleckne@elon.edu \\ Shannon Duvall, Elon University, sduvall2@elon.edu
}

\begin{abstract}
Digital storytelling is the use of multimedia presentations to convey information in a way that is logical, yet often deeply emotional. Through the use of global media like images and music, stories can be told that span language, culture, and disciplinary boundaries. In this paper, we describe the ways that digital storytelling can be used in the classroom, both as a learning assessment method and as a teaching tool. We show how the use of digital storytelling in classes can make computing skills relevant in a noncomputing class, as well as attract non-technical students to the computing field.
\end{abstract}

Keywords: digital storytelling, digital technologies, general studies, multimedia, pedagogy, visualization.

\section{INTRODUCTION}

Storytelling has been around since the beginning of time. It "is an ancient art rooted in our common human culture, as well as in our physiology and psychology" [4]. Digital storytelling, however, is fairly new, especially in the classroom. A digital story is "a three to four minute digital multimedia 'movie' that combines an original story or script with images, music, and above all, a narration in the author's own voice" [18]. Stories often elicit strong responses from the listeners and require deep understanding from the creator.

Unlike essays or bulleted lists, digital storytelling often hinges on tying together otherwise disconnected statements into an overarching plot and creating an emotional response in the listener. Statements must be backed up to be convincing and believable. For these reasons, we believe digital storytelling to be a good assessment device for student learning. In addition, the students enjoy being able to add a creative element to their projects. They get to show what they have learned, but with their own perspective and in their own voices.
Instructors can also use digital storytelling to present new material to the class. The use of visual and auditory cues is appealing to students, and the story attaches an experience to the concepts being conveyed, which aid in remembering them later. The use of storytelling to teach is especially important in a technical field. Presenting technical material in a non-technical metaphor makes the class more concrete and memorable, especially for students who are traditionally turned-off by technical concepts.

In addition, the story products, whether created as teaching tools by professors or learning assessments by students, are the means for communicating across disciplines and cultures. Stories created through visuals can even cross language barriers. Visualizing information "communicates to a global audience" [8].

In this paper, we present the use of digital storytelling by both teachers and students in the classroom. We begin with an overview of the known advantages of the use of storytelling. We then present our methods for the use of digital storytelling and why it should be more widely used. Finally, we point out possible pitfalls with the storytelling model.

\section{ADVANTAGES OF STORYTELLING}

Storytelling is being used across many disciplines. "A well-told story conveys great quantities of information in relatively few words in a format that is easily assimilated by the listener or viewer. People usually find it easier to understand information integrated into stories than information spelled out in serial lists (such as bulleted items in an overhead slide). Stories are also just more compelling” [4].

Likewise, digital storytelling is "transforming our classrooms from spaces of discovery to spaces of active inquiry and authorship" [18]. Weis [18] shares examples of using storytelling with students in an African American History class as well as a Latina Life Stories class. Digital storytelling allows the students to synthesize their own stories and research collaboratively. Weis finds that through "digital presentations, students become more conscious of, 
and reflective about, the power and responsibilities of historical synthesis and interpretation” [18].

Companies are even using digital storytelling. PricewaterhouseCoopers and Coca Cola are using storytelling for marketing motivation, and communication [9]. "Global companies are recognizing visual information as a method to unite and communicate with other cultures" [8]. Language and cultural barriers are minimized through visualizing information. Students at Rochester Institute of Technology studied utilizing basic strategies for displaying complex data [8]. They found that visual resources such as tables, diagrams, charts and maps were creative solutions for potentially difficult communication problems.

McLellan [9] recognizes that "design, research, and theory-building in several diverse fields have found a common goal in the quest to design experiences that serve some purpose in a compelling and memorable way. Many well-tested design ideas from outside of education, from a wide range of fields and applications, offer great potential for the design of online classes and university campuses in cyberspace.”

Storytelling is becoming an accepted practice in the classroom. Whatever the use of visuals in the classroom, as Burns [2] notes, "students today are quite media literate". By creating and watching digital stories, students of all ages can benefit through learning, motivation, and active engagement.

\section{A NEW MODEL}

Digital storytelling is a powerful bridge between Information Technology (IT) and Arts and Humanities courses. In some disciplines, most notably History, storytelling is the most fundamental pedagogical tool. Digital storytelling can be introduced as a way for students to take their multimedia authoring skills into these Arts and Humanities classes to give a deeper, interdisciplinary learning experience.

In addition, we adopt digital storytelling as a teaching tool in the IT classroom. By telling stories to introduce computing concepts, we provide a new way to present material that appeals to non-technical students who may otherwise be turned off to computing. In doing so we also are more effective in conveying material to any in the class who learn visually or through metaphor.
Instructive Approach. Digital storytelling can be used as a teaching tool in the classroom. In a time when there are severely underrepresented minority groups in computing, it is vitally important to find ways to reach out to an audience not traditionally attracted to computing. Digital storytelling is one means to bridge this gap.

Stories can be used in the IT classroom to introduce new concepts at the beginning of class. The storytelling need not be a time-consuming part of class. Those in the class that learn through metaphor will understand the "meaning" of the story by the end of the class with no further explanation. Others may not pick up on the more subtle parallels of the story to the computing concepts, but it is unnecessary (and in our opinion, unadvisable) to point them out. We do not test on the stories nor require their understanding, and therefore the only class time spent on the stories is in reading them.

Several popular children's books by Dr. Seuss can be used to introduce concepts in a beginning programming course. We use "Too Many Daves" [15] to introduce arrays, "The Cat in the Hat Comes Back" [12] to teach recursion, "King Looie Katz" [13] for linked lists, "Yertle the Turtle" [16] to teach stacks, and "The Lorax" [14] for deadlocking. While these are traditional rather than digital stories, they are readily available resources and have the advantage that students are familiar already with many of the stories. Students who traditionally feel intimidated because they have no prior programming experience learn that they have been exposed to some fundamental computing concepts after all.

In addition, we have written several digital stories for direct metaphors for programming concepts. In "Tarzan, Variable of the Jungle," students learn variable scope through a family of variables who are not allowed to immigrate to other lands or even go on vacation. The story, told through narration and images, is meant to help the students remember the syntax rules for variable scope. The story provides a shared vocabulary and experience for all the students in the class. We once overheard a pair of students in the lab debugging syntax errors. One student noticed the error in variable scope and exclaimed to his partner, "Oh! Variables born in Methodpotamia must stay in Methodpotamia!" The story not only provided the mental reminder of the concept covered in class, but gave the student a non-technical way to refer to it.

Overall, students find "story time" a fun part of class and routinely ask for them. When polled about the 
stories, all but one student said that some story helped in their understanding and memory of the concepts being introduced. (The student's response for all stories was "Neutral.") Only one student disagreed with the statement, "I found the stories to be a fun part of class" and, interestingly, this student had a computing-related major and found all of the stories helpful in learning and understanding concepts. All students surveyed agreed with the statement "If I were to take the class again, I would like the stories to be a part of the course." One student also commented, "The stories were a cute/fun way to help further my understanding (or clear up some confusions) of certain topics. I would suggest they be used in your next class."

Learning Approach. In addition to telling the students stories, we also require the students exhibit their learning by creating stories. As a pedagogical tool, digital storytelling gives the students a powerful means of communicating and in turn forces a deeper learning experience. Students must go through the process of reflecting on what is important to communicate about their topic. It is noted that "while not every student is an aspiring Speilberg, most students can gain tremendous insight into storytelling by delving into the creation process" [3]. The process forces a greater rigor in deciding what to show and what to tell.

A non-IT course was taught on global studies using two types of student projects. The students were first asked to write a research paper on an environmental issue with the goals of conducting scholarly research, discovering global issues on their own, and articulating how they relate to the rest of the world.

The students were then asked to pair up and create a digital story using one of their environmental issues. The main goal of the digital story project was to take their research paper and evolve it into an emotive project with a heavy visual component. In class we had already discussed the relationship between verbal and visual ways of constructing knowledge.

The digital story projects relied on the use of IT tools such as PowerPoint or iMovie, yet the assignment was not graded based on their technical proficiency. Both projects were graded on purpose, organization, content, and persuasiveness. The digital stories were much more compelling and the students enjoyed the second project more. Not surprisingly, grades were also higher.

A digital story usually begins with a script. Students then must engage in active inquiry to collect images to support the ideas and emotions in the script. Once the images are assimilated, the student narrates the script, combining voice and music to offer an affective story.

Digital stories allow students to express themselves with their own words and voices, fostering a sense of authorship. This sense of individuality and ownership ultimately generates a much deeper experience for the student. The end result is also a much richer product.

For many students, visuals offer a different mode of conveying information. It is worth noting the different roles that images can play. Figures 1-4 are samples from student work.

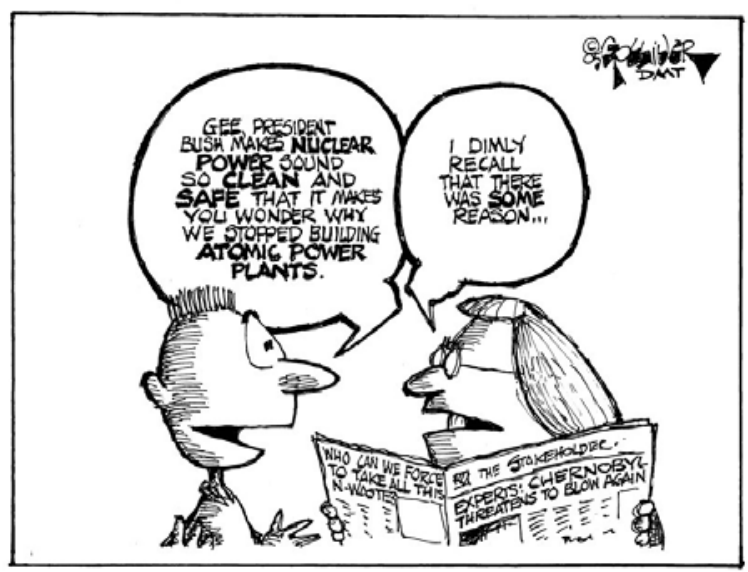

Figure 1: This comic was used as an attention grabber [4]. Comic situations can often add to the story.

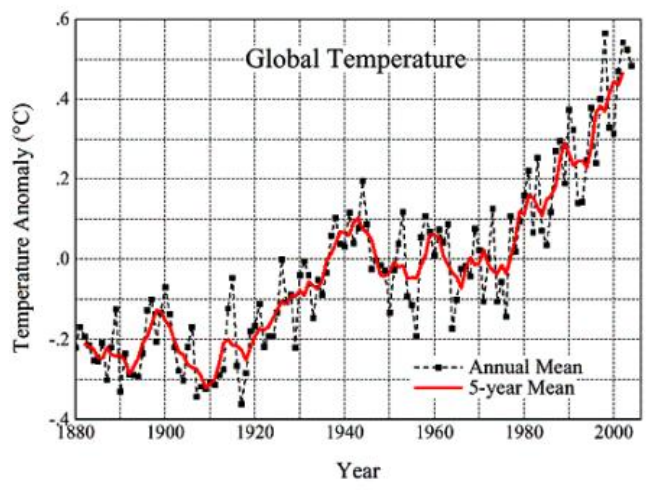

Figure 2: This chart was used to support scientific data [11]. Students can either create their own charts, or find them online. 


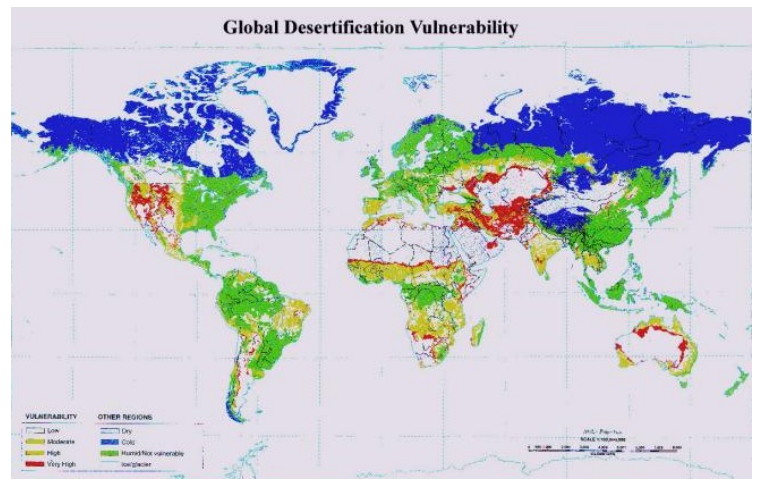

Figure 3: As shown with this image, maps can also be found to support research [6]. More often, maps are used to convey visual reference.

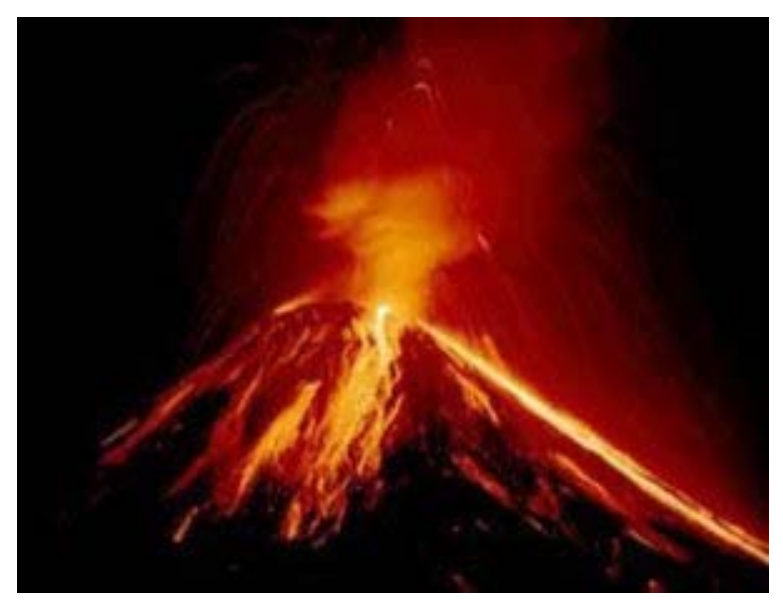

Figure 4: Powerful images, such as this one, can be used for persuasive storytelling [17]. Unique and powerful images are best for evoking emotion.

\section{ISSUES IN STORY CREATION}

Compelling resources alone won't create a compelling story. However, a few key images mixed with voice and emotive music can convey an inspiring story. Steps in building a digital story include creating a script, collecting images, narrating the story, and finding emotive music.

When creating a story to be used in a class setting, it is important to be aware of subtle messages being sent by the story. Since it is easy to inadvertently create a biased view, use a stereotype, or state an opinion in a story, it is important to reread all stories looking for inappropriate messages. Raising politically or socially charged issues will distract from the lesson and should be avoided.
In using stories in class such as the Dr. Seuss stories, we are careful to tell the students that we do not intend to "talk down" to them or trivialize the concepts we are trying to teach. We explain our reasoning for the stories and the goal of making class more interesting, memorable, and fun. We have not had any negative response to our storytelling, and we believe it is in part due to our willingness to talk openly with the students about our methods.

When charging students with creating a story, the same precautions about unwanted subtle messages apply. The professors must be careful to create a standard the stories must meet. Rubrics should be used to lay out these standards for the students. Rubrics also aid technical professors who find it difficult to grade creative work.

In addition, students should be sure they follow appropriate copyright laws and are aware of the ethical issues surrounding intellectual property. It is rarely the case that the students are generating completely original images and music, and they must be educated in how to site their references.

No matter how technology savvy the students may be, technology issues may arise when creating digital stories. In the creation process, students must import music, record their voices, and compose and edit their stories. Although daunting, this process should be clearly defined and the technology aspect should be considered secondary to the storytelling.

\section{CONCLUSION}

Digital storytelling is a compelling means for conveying information. It can be used in any classroom - including the computing classroom - to introduce material as well as assess student learning. Digital stories make material compelling, interesting, memorable, and accessible to a larger audience. In any discipline, students can learn through digital storytelling and can learn the skill of telling stories of their own. Digital storytelling should therefore be considered as an alternative teaching tool. After all, "we live in a visual world" [8].

\section{REFERENCES}

1. Bouchlaghem, N., Wilson, A., Beacham, N., and Sher, W. (2002). Computer Imagery and Visualization in Built Environment Education: The CAL-Visual Approach. Innovations in 
Education and Teaching International, 39(3), 225-236.

2. Burns, M., Dimock, V., \& Martinez, D. (2000). How Can Technology Help in the Developmental Process? TAP into Learning. 2/3, 6.

3. Burns, M., Dimock, V., \& Martinez, D. (2000). Storytelling in a Digital Age. TAP into Learning. $3 / 2,20-22$.

4. (2007). Carolina Peace Resource Center. Retrieved March 6, 2007, from Carolina Peace Web site: http://carolinapeace.org/

5. Gershon, N., \& Page, W. (2001). What Storytelling Can Do for Information Visualization. Communications of the ACM. 44/8, 31-37.

6. (2007). GFZ Potsdam. Retrieved March 6, 2007, from GeoForschungsZentrum Potsdam Web site: http://www.gfz-potsdam.de/

7. Green, D. (2006).Using Digital Images in Teaching and Learning: Perspectives from Liberal Arts Institutions. Academic Commons.

8. Jackson, C., \& Ciolek, N. (2001). Designing Visual Information for a Global Audience. International Conference on Computer Graphics and Interactive Techniques.

9. McLellan, H. (2000).Experience Design. CyberPsychology \& Behavior. 3/1, 59-69.
10. Pollard, S., \& Duvall, R.C. (2006). Everything I Needed to Know About Teaching I Learned in Kindergarten: Bringing Elementary Education Techniques to Undergraduate Computer Science Classes. Proceedings of the Thirty-Seventh SIGCSE Technical Symposium on Computer Science Education. 224-228.

11. Schmunk, Robert B. GISS Surface Temperature Analysis. Retrieved March 6, 2007, from Data @ NASA GISS Web site: http://citationmachine.net/

12. Seuss, D. (1958). The Cat in the Hat Comes Back. Random House.

13. Seuss, D. (1969). I Can Lick 30 Tigers Today And Other Stories. Random House.

14. Seuss, D. (1971). The Lorax. Random House.

15. Seuss, D. (1961). Sneetches and Other Stories. Random House.

16. Seuss, D. (1958). Yertle the Turtle. Random House.

17. (2007). Volcano Discovery. Retrieved March 6, 2007, from Volcano Discovery Tours Web site: http://www.volcanodiscovery.com/volcanotours/photos/volcanoes/

18. Weis, T.M., Benmayor, R., O'Leary, C., \& Eynon, B. (2002). Digital Technologies and Pedagogies. Social Justice, 29(4), 153-167. 